\title{
QCD STUDIES WITH W AND Z BOSONS AT THE TEVATRON*
}

\author{
GEORG STEINBRÜCK \\ (FOR THE CDF AND D $\emptyset$ COLLABORATIONS) \\ Columbia University, Nevis Labs, 136 S. Broadway, Irvington NY 10533, USA \\ E-mail: steinbru@fnal.gov
}

\begin{abstract}
The CDF and D $\varnothing$ experiments have both collected large samples of $W$ and $Z$ bosons with the last Tevatron collider run (1995-1996) using $p \bar{p}$ collisions at $\sqrt{s}=$ $1.8 \mathrm{TeV}$. We present the results of QCD studies with vector bosons that cover a large range of transverse momentum space, $p_{T}$, making this a testing ground for both perturbative and non-perturbative QCD. The measurement of the $W$ and $Z$ cross section, their width, the $W$ and $Z$ transverse momentum distribution and the angular distribution of electrons in $W$ decays are described in this paper.
\end{abstract}

\section{Introduction}

At the Fermilab Tevatron, $W$ and $Z$ bosons are produced in high energy $\bar{p} p$ collisions. The study of the production of $W$ and $Z$ bosons provides an avenue to explore QCD, the theory of strong interactions. The benefits of using intermediate vector bosons to study perturbative QCD are large momentum transfer, distinctive event signatures, low backgrounds, and a well understood electroweak vertex. In this paper we present several measurements of $W$ and $Z$ boson properties based on data taken by the $\mathrm{D} \varnothing$ and CDF collider detectors during the 1992-1993 (Run 1A) and 1994-1996 (Run 1B) Tevatron running periods.

In the parton model at lowest order, $W$ and $Z$ intermediate vector bosons are produced in head-on collisions of $q \bar{q}$ constituents of the proton and antiproton, and have little transverse momentum $\left(p_{T}<<M_{W}, M_{Z}\right)$. Consequently, the fact that observed bosons have large transverse momentum $\left(p_{T}\right)$ is attributed to the production of one or more gluons or quarks along with the bosons. As a result, QCD corrections become important and modify electroweak processes: Bosons are produced with an average transverse momentum of $\approx 10 \mathrm{GeV}$. Boson + jet events are possible where $W+1$ jet events occur $\approx 7 \%$ of the time for $E_{T}^{\text {jet }}>25 \mathrm{GeV}$. The inclusive production cross sections for $W$ and $Z$ bosons are enhanced by a $\mathrm{K}$ factor of $\approx 18 \%$, and the angular distribution of decay electrons in $W$ boson events is modified when QCD effects are taken into account.

* PAPER PRESENTED AT THE LAKE LOUISE WINTER INSTITUTE, LAKE LOUISE, ALBERTA, CANADA, 20-26 FEBRUARY 2000. 


\section{The $W$ and $Z$ Inclusive Production Cross Sections}

New results on the $W$ and $Z$ production cross sections times electronic branching ratios from $\mathrm{CDF}$ and $\mathrm{D} \varnothing$ are shown in Fig. 1. DØ measure ${ }^{1}$ $\sigma_{W} \cdot B(W \rightarrow e \nu)=2310 \pm 10$ (stat) \pm 50 (syst) \pm 100 (lum) pb and $\sigma_{Z} \cdot B\left(Z \rightarrow e^{+} e^{-}\right)=221 \pm 3$ (stat) \pm 4 (syst) \pm 10 (lum) pb, where "lum" is due to the uncertainty on the integrated luminosity. CDF obtain ${ }^{2} \sigma_{Z} \cdot B\left(Z \rightarrow e^{+} e^{-}\right)=249 \pm 5$ (stat $\oplus$ syst) \pm 10 (lum) pb and $\sigma_{Z} \cdot B\left(Z \rightarrow \mu^{+} \mu^{-}\right)=237 \pm 9$ (stat $\oplus$ syst) \pm 9 (lum) pb.

The errors are dominated by the uncertainty in the integrated luminosity of the data samples. Note, that D $\varnothing$ and CDF use different total $p \bar{p}$ cross sections to determine their integrated luminosities. CDF use their own measurement ${ }^{3}$, while D $\varnothing$ take the average of the CDF, E710 ${ }^{4}$ and E811 ${ }^{5}$ measurements. To properly compare the measured cross sections, the DØ Run $1 \mathrm{~b}$ cross sections must be multiplied by 1.062 if using the CDF normalization.

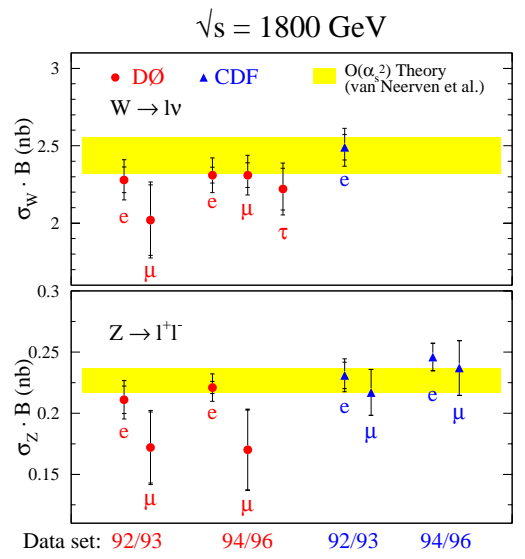

Figure 1. Measurements of the $W \rightarrow e \nu$ and $Z \rightarrow e^{+} e^{-}$cross sections from D $\varnothing$ and CDF.

\section{Extraction of the Width of the $W$ Boson from the Ratio of $W \rightarrow e \nu$ and $Z \rightarrow e^{+} e^{-}$Cross Sections}

The integrated luminosity uncertainty and many of the other systematic errors cancel in the ratio of cross sections $R=\sigma_{W} \cdot B(W \rightarrow e \nu) / \sigma_{Z} \cdot B\left(Z \rightarrow e^{+} e^{-}\right)$. This allows indirect, precise measurements of the $W \rightarrow e \nu$ branching fraction and the width of the $W$ boson. This follows using

$$
\frac{\sigma_{W} \cdot B(W \rightarrow e \nu)}{\sigma_{Z} \cdot B\left(Z \rightarrow e^{+} e^{-}\right)}=\frac{\sigma_{W}}{\sigma_{Z}} \frac{1}{B\left(Z \rightarrow e^{+} e^{-}\right)} \frac{\Gamma(W \rightarrow e \nu)}{\Gamma(W)}
$$


together with the theoretical calculation of $\sigma_{W} / \sigma_{Z}{ }^{6}$, the measured $Z \rightarrow e^{+} e^{-}$ branching ratio from LEP ${ }^{7}$, and the SM value of $\Gamma(W \rightarrow e \nu)^{8}$.

The measured values of $R$ are $R=10.49 \pm 0.14$ (stat) \pm 0.21 (syst) for $\mathrm{D} \varnothing$ and $R=10.38 \pm 0.14$ (stat) \pm 0.17 (syst) for CDF, using the combined electron data from Runs $1 \mathrm{a}$ and $1 \mathrm{~b}$. The main sources of systematic errors are due to uncertainties in backgrounds, efficiencies, and electron energy scale. A $1 \%$ error due to NLO electroweak radiative corrections ${ }^{1}$ is also included. The two $R$ measurements have been combined, yielding $R=10.42 \pm 0.18$. Using this combined value of $R$, the resulting branching fraction is $B(W \rightarrow$ $e \nu)=(10.43 \pm 0.25) \%$ and the width of the $W$ boson is determined to be $\Gamma(W)=2.171 \pm 0.052 \mathrm{GeV}$. The results agree with the SM predictions when the errors are taken into account, as shown in Fig. 3.

A direct measurement of the $W$ boson width is possible using a fit to transverse mass $\left(M_{T}\right)$ spectrum in $W$ events (see Fig. 2). The $W$ width directly affects the shape of the distribution, most prominently at high values of $M_{T}$, where the Breit-Wigner line shape dominates over detector resolution effects. CDF have new preliminary results for Run $1 \mathrm{~b} W \rightarrow e \nu$ and $W \rightarrow \mu \nu$ events, using a binned likelihood fit in the region $M_{T}>100 \mathrm{GeV} / c^{2}$. The $W$ events are modeled using a similar simulation to that used in the $W$ mass analysis. This method is less model-dependent than the indirect measurement discussed above, but with the current data sets it is statistically limited. The results are $\Gamma(W)=2.17 \pm 0.125$ (stat) \pm 0.105 (syst) $\mathrm{GeV}$ from the electron data and $\Gamma(W)=1.78 \pm 0.195$ (stat) \pm 0.135 (syst) GeV from the muon data. These results are combined with the Run 1a electron measurement, yielding $\Gamma(W)=2.055 \pm 0.100$ (stat) \pm 0.075 (syst) GeV. This result is consistent with the SM prediction, as shown in Fig. 3.
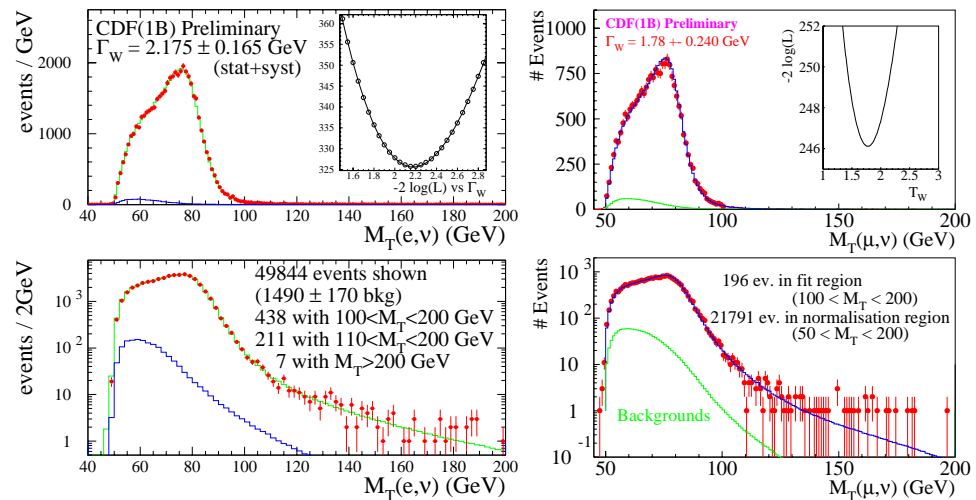

Figure 2. CDF direct measurement of the $W$ boson width using a fit to the $e \nu$ (left) and $\mu \nu$ transverse mass (right). 

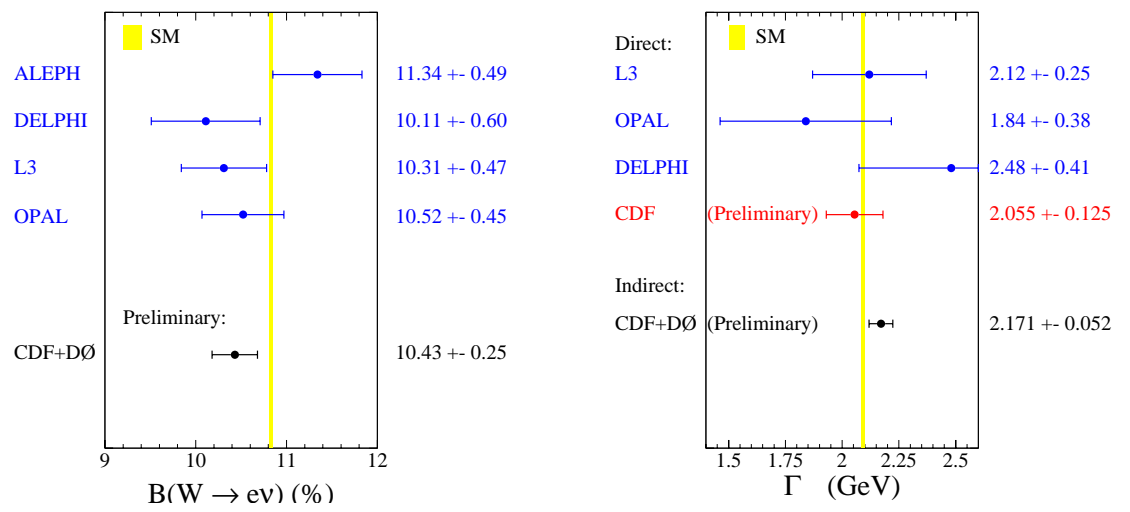

Figure 3. Measurements of $B(W \rightarrow e \nu)$ (left) and summary of direct and indirect measurements of the $W$ width (right).

\section{The $W$ and $Z$ Transverse Momentum Distributions}

Three regions of transverse momentum space can be distinguished: At high transverse momentum $\left(p_{T}>20 \mathrm{GeV} / c\right)$, where the cross section is dominated by the radiation of a single parton with large transverse momentum, perturbative QCD is expected to be reliable ${ }^{9}$. At low transverse momentum $\left(p_{T}<10 \mathrm{GeV} / c\right)$, multiple soft gluon emission is expected to dominate the cross section. and a soft gluon resummation technique ${ }^{10,11}$ is used to make QCD predictions. When $p_{\mathrm{T}}$ approaches $\Lambda_{Q C D}$, however, non-perturbative aspects of the strong force become dominant and even resummed calculations fail. A parameterization is used to extend the calculation to the very low $p_{T}$ region. The parameters are obtained from fits to low energy Drell-Yan data 10,11 .

Here we present the measurements of the $W$ and $Z$ boson $p_{T}$ spectra using electronic decay modes. The measured $W$ and $Z p_{T}$ distributions are corrected for known backgrounds bin-by-bin. D $\varnothing$ 's result for the $W p_{T}$ distribution ${ }^{12}$, shown in Fig. 4 (left), is compared to the theoretical calculation by Arnold and Kauffman ${ }^{10}$, smeared by detector resolutions. The $W$ data shows good agreement with this combined QCD perturbative and resummation calculation over the whole range of $p_{T}$. In the case of the $Z, C D F$ and $D \varnothing$ correct the measured cross section for the effects of detector smearing. Fig. 4 (right) shows CDF's smearing-corrected $Z p_{T}$ measurement ${ }^{13}$ compared to the calculation by Ladinsky and Yuan ${ }^{11}$. Fig. 5 (left) compares DØ's final, smearingcorrected $Z p_{T}$ distribution ${ }^{14}$ to the calculation by Ladinsky and Yuan ${ }^{11}$. 
Shown on the right is the fractional difference between the $Z$ data and the combined calculation and the fixed-order perturbative theory ${ }^{9}$, respectively. In comparing to the NLO calculation, D0 observe strong disagreement at low $p_{T}$, as expected due to the divergence of the calculation at $p_{T}=0$, and a significant enhancement of the cross section from soft gluon emission.
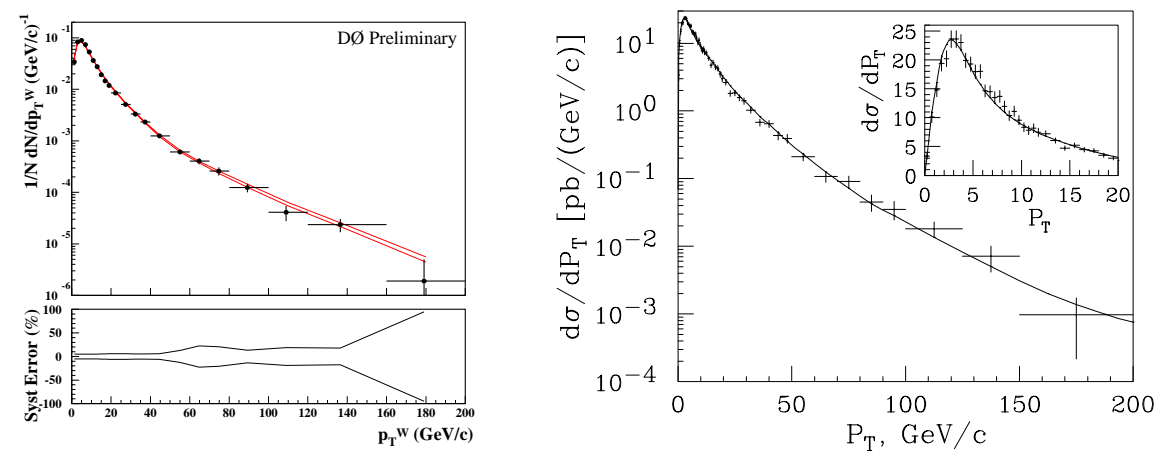

Figure 4. Left: $\mathrm{D} \varnothing W p_{T}$ result (solid points) with statistical uncertainty. The theoretical calculation by Arnold-Kauffman has been smeared for detector resolutions and is shown as two lines corresponding to the $\pm 1 \sigma$ variations of the uncertainties in the detector modeling. Data and theory are independently area normalized to unity. The fractional systematic uncertainty on the data is shown as a band in the lower portion of the plot. Right: The CDF $Z p_{T}$ smearing-corrected data (crosses) compared to the combined resummed and NLO perturbative prediction normalized to the data (curve).
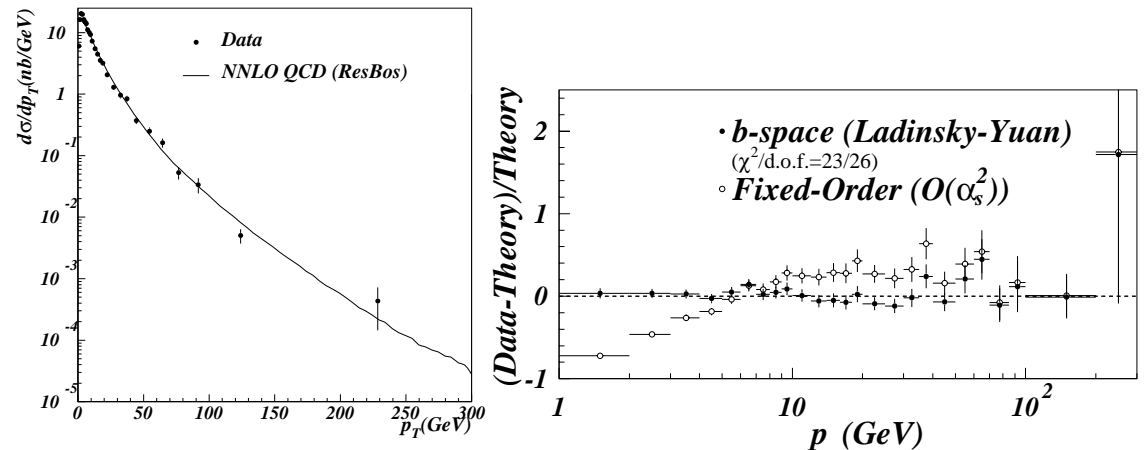

Figure 5. Left: $\mathrm{D} \varnothing Z p_{T}$ smearing-corrected data (solid points) with total uncertainty shown compared to the combined resummed and NLO perturbative prediction. The data is normalized to the $\mathrm{D} \varnothing$ measured inclusive $Z$ production cross section; the theory is normalized to its own prediction. Right: Fractional difference between the $Z$ data and the resummed and fixed-order calculation, respectively, as a function of $p_{T}$. 


\section{The Angular Distribution of Electrons in $W$ Boson Decays}

A new measurement of the electron angular distribution parameter $\alpha_{2}$ in $W \rightarrow e \nu$ events by $\mathrm{D} \varnothing$ is presented here ${ }^{15}$. Our results are compared with next-to-leading order perturbative QCD, which predicts an angular distribution of $\left(1 \pm \alpha_{1} \cos \theta^{*}+\alpha_{2} \cos ^{2} \theta^{*}\right)^{16}$, where $\theta^{*}$ is the polar angle in the Collins-Soper frame ${ }^{17}$. In the presence of QCD corrections, the parameters $\alpha_{1}$ and $\alpha_{2}$ become functions of $p_{T}^{W}$, the $W$ boson transverse momentum. We present the first measurement of $\alpha_{2}$ as a function of $p_{T}^{W}$. This measurement is of importance, because it provides a test of next-to-leading order QCD corrections which are a non-negligible contribution to the $W$ mass measurement.

Due to only being able to measure the transverse components of the neutrino momentum, the transformation from the lab frame to the $W$ rest frame (Collins-Soper frame) is not calculable. Therefore the polar angle of the electron from $W$ decay, $\theta^{*}$, is not directly measurable. In this analysis, the electron angle $\theta^{*}$ is inferred from the correlation between the transverse mass of the $W\left(M_{T}^{W}\right)$ and $\cos \theta^{*}$ through the use of Bayes Theorem ${ }^{18}$ (see Fig. 6 (left)).

Therefore, we calculate the the probability of measuring $M_{T}^{W}$ for a given value $\cos \theta^{*}$ in a given $p_{T}^{W}$ bin; $g\left(\cos \theta^{*} \mid M_{T}^{W}\right)$. This probability function is inverted to give the probability of measuring $\cos \theta^{*}$ for a measured $M_{T}^{W}$, $f\left(\cos \theta^{*} \mid M_{T}^{W}\right)$, using Bayes theorem:

$$
f\left(\cos \theta^{*} \mid M_{T}^{W}\right)=\frac{g\left(M_{T} \mid \cos \theta^{*}\right) h\left(\cos \theta^{*}\right)}{\int g\left(M_{T} \mid \cos \theta^{*}\right) h\left(\cos \theta^{*}\right) d \cos \theta^{*}}
$$

where $h\left(\cos \theta^{*}\right)$ is the prior probability function, which we take to be $h\left(\cos \theta^{*}\right)=\left(1+\cos ^{2} \theta^{*}\right)$, the expectation from $V-A$ theory without QCD modification.

To derive the probability function, $g\left(M_{T} \mid \cos \theta^{*}\right)$, we use a Monte Carlo simulation of the $\mathrm{D} \varnothing$ detector. After determining $g\left(M_{T} \mid \cos \theta^{*}\right)$, it is inverted, and the angular distribution is calculated in four $p_{T}^{W}$ bins. With the unfolded angular distributions now calculated, the value of $\alpha_{2}$ in each of the four $p_{T}^{W}$ bins can be determined. This is accomplished by generating a set of angular distribution templates in Monte Carlo for different values of $\alpha_{2}$. The templates are then compared to the data through the use of a maximum log-likelihood. Fig. 6 (center) shows the measured angular distribution with three templates for different values of $\alpha_{2}$ and $20 \mathrm{GeV}<p_{T}^{W}<35 \mathrm{GeV}$. The results of our measurement along with the theoretical prediction are given in Fig. 6 (right). The QCD prediction is preferred by $\approx 2.3 \sigma$ over a $(V-A)$ theory without QCD effects taken into account.

FERMILAB-CONF-00/094-E 

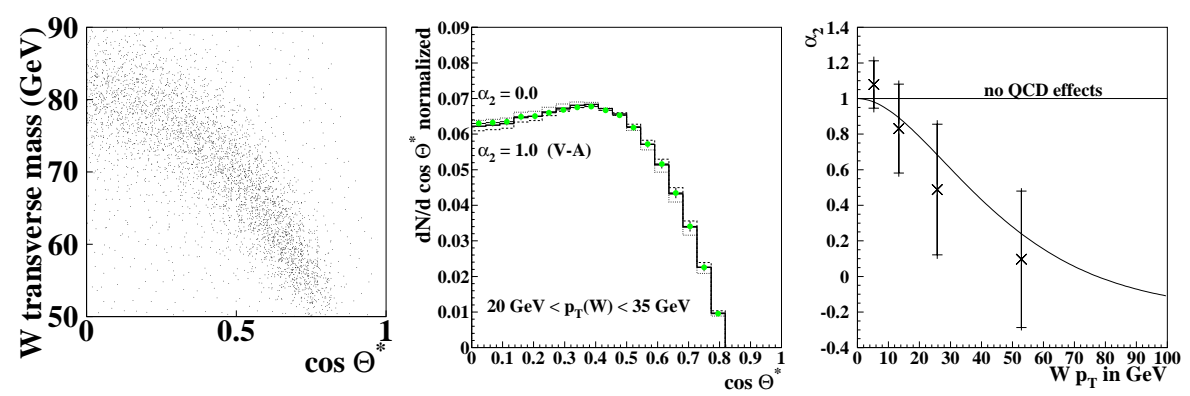

Figure 6. Left: Smeared $W$ transverse mass versus true $\cos \theta^{*}$ for $p_{T} \leq 10 \mathrm{GeV}$ (acceptance cuts applied). Center: Measured angular distributions compared to Monte Carlo templates for $20 \mathrm{GeV}<p_{T}<35 \mathrm{GeV}$. Shown is the template that fit best (solid) and the templates for $\alpha_{2}=1.0$ (dashed) and $\alpha_{2}=0.0$ (dotted). The drop-off at small angles is due to limited acceptance. Right: Measured $\alpha_{2}$ as a function of $p_{T}$ compared to NLO QCD calculation by Mirkes (curve) and calculation in the absence of QCD (horizontal line). The vertical bars denote the total errors while the statistical errors are marked by horizontal ticks.

\section{References}

1. B. Abbott et al. (DØ collaboration), Phys. Rev. D 61, 072001 (2000).

2. F. Abe et al. (CDF collaboration), Phys. Rev. D 59, 052002 (1999).

3. F. Abe et al. (CDF collaboration), Phys. Rev. D 50, 5550 (1994).

4. N. Amos et al. (E710 collaboration), Phys. Rev. Lett. 68, 2433 (1992).

5. C. Avila et al. (E811 collaboration), Phys. Lett. B 445, 419 (1999).

6. R. Hamberg, W.L. van Neerven, T. Matsuura, Nucl. Phys. B359, 343 (1991); W.L. van Neerven, E.B. Zijlstra, Nucl. Phys. B382, 11 (1992).

7. L. Montanet et al., (Particle Data Group), Phys. Rev. D 54, 1 (1996).

8. L. Rosner, M.P. Worah, and T. Takeushi, Phys. Rev. D 49, 1363 (1994).

9. P.B. Arnold and M.H. Reno, Nucl. Phys. B319, 37 (1989); B330, 284E (1990). P.B. Arnold and R.P. Kauffman, Nucl. Phys. B349, 381 (1991).

10. P.B. Arnold and R.P. Kauffman, Nucl. Phys. B349, 381 (1991).

11. G.A. Ladinsky and C.-P. Yuan, Phys. Rev. D 50, 4239 (1994).

12. B. Abbott et al. (DØ collaboration), hep-ex/9907044, paper submitted to EPS-HEP 99; Tampere, Finland, July 1999.

13. T. Affolder et al. (CDF collaboration), Phys. Rev. Lett. 84, 845 (2000).

14. B. Abbott et al. (DØ collaboration), Phys. Rev. D 61, 032004 (2000); Phys. Rev. Lett. 84, 2792 (2000).

15. B. Abbott et al. (D Ø collaboration), hep-ex/9907022, paper submitted to EPS-HEP 99; Tampere, Finland, July 1999 and G. Steinbrück, Ph.D. Thesis, The University of Oklahoma, 1999 (unpublished).

16. E. Mirkes, Nuclear Physics, B387 3 (1992).

17. J.C. Collins, D.E. Soper, Phys. Rev. D 16,2219, (1977).

18. E. T. Jaynes, "Probability Theory: The Logic of Science", unpublished. 